Article

\title{
Evaluation of Anti-Mycobacterial Compounds in a Silkworm Infection Model with Mycobacteroides abscessus
}

\author{
Kanji Hosoda ${ }^{1,2}$, Nobuhiro Koyama ${ }^{1,2}{ }^{-}$, Hiroshi Hamamoto ${ }^{3}\left(\mathbb{D}\right.$, Akiho Yagi $^{4}$, Ryuji Uchida ${ }^{4}$, \\ Akihiko Kanamoto ${ }^{5}$ and Hiroshi Tomoda ${ }^{1,2, *}$ \\ 1 Department of Microbial Chemistry, Graduate School of Pharmaceutical Sciences, Kitasato University, \\ Tokyo 108-8641, Japan; hosodak@pharm.kitasato-u.ac.jp (K.H.); koyaman@pharm.kitasato-u.ac.jp (N.K.) \\ 2 Medicinal Research Laboratories, School of Pharmacy, Kitasato University, Tokyo 108-8641, Japan \\ 3 Institute of Medical Mycology, Teikyo University, Tokyo 192-0395, Japan; hamamotoh@main.teikyo-u.ac.jp \\ 4 Faculty of Pharmaceutical Sciences, Tohoku Medical and Pharmaceutical University, Sendai 981-8558, Japan; \\ 21752501@is.tohoku-mpu.ac.jp (A.Y.); uchidar@tohoku-mpu.ac.jp (R.U.) \\ 5 OP Bio Factory Co. Ltd., Okinawa 904-2234, Japan; akihiko.kanamoto@opbio.com \\ * Correspondence: tomodah@pharm.kitasato-u.ac.jp
}

Received: 2 October 2020; Accepted: 26 October 2020; Published: 27 October 2020

\begin{abstract}
Among four mycobacteria, Mycobacterium avium, M. intracellulare, M. bovis BCG and Mycobacteroides (My.) abscessus, we established a silkworm infection assay with My. abscessus. When silkworms (fifth-instar larvae, $n=5$ ) were infected through the hemolymph with My. abscessus $\left(7.5 \times 10^{7} \mathrm{CFU} /\right.$ larva) and bred at $37^{\circ} \mathrm{C}$, they all died around $40 \mathrm{~h}$ after injection. Under the conditions, clarithromycin and amikacin, clinically used antimicrobial agents, exhibited therapeutic effects in a dose-dependent manner. Furthermore, five kinds of microbial compounds, lariatin A, nosiheptide, ohmyungsamycins A and B, quinomycin and steffimycin, screened in an in vitro assay to observe anti-My. abscessus activity from 400 microbial products were evaluated in this silkworm infection assay. Lariatin A and nosiheptide exhibited therapeutic efficacy. The silkworm infection model with My. abscessus is useful to screen for therapeutically effective anti-My. abscessus antibiotics.
\end{abstract}

Keywords: silkworm; mycobacteria; nontuberculous mycobacteria; Mycobacterium avium complex; Mycobacteroides abscessus; antimycobacterial activity; microbial product; lariatin; nosiheptide; ohmyungsamycin; steffimycin; quinomycin

\section{Introduction}

In the process of antibiotic discovery, candidate compounds active against pathogenic microorganisms in an in vitro assay system often have no therapeutic effects in in vivo animal infection models. Therefore, therapeutic efficacies of candidate compounds in an in vivo assay need to be evaluated at the early stage of drug development. However, in vivo evaluation using mice, rats or rabbits is time-consuming and expensive, in addition to having ethical issues. In order to overcome these issues, we established in vivo-mimic infection assays using silkworms (fifth-instar larvae) with methicillin-resistant Staphylococcus aureus (MRSA) [1-4], Pseudomonas aeruginosa and Candida albicans [5-8]. Thus, the silkworm infection model has many advantages over the mouse infection model such as fewer ethical issues, lower maintenance costs, less space required to keep animals, less drugs required for evaluations and shorter times for infection experiments [6,7].

Pulmonary diseases caused by non-tuberculous mycobacteria (NTM) are increasing worldwide. Of note, in several areas, including the United States, Canada and Japan, the incidence rate of NTM disease is higher than that of tuberculosis (TB) [9-11]. The major causative agents of NTM diseases are 
Mycobacterium avium, $M$. intracellulare (a mixed infection with $M$. avium and $M$. intracellulare is called Mycobacterium avium complex (MAC)) and Mycobacteroides (My.) abscessus for more than $90 \%$ of the patients with NTM disease. Although clarithromycin (CAM), rifampicin (RFP) and ethambutol (EB) are used for pulmonary NTM infection, their therapeutic effects are limited [12,13]. Therefore, it is currently important to discover new drugs for the treatment of NTM infection. Accordingly, we started to search for new microbial antibiotics active against NTM. In the present study, we first found that lariatins [14], nosiheptide [15,16], ohmyungsamycins [17], steffimycin [18,19] and quinomycin A [20] exhibited in vitro anti-mycobacterial activity against $M$. avium, M. intracellulare and My. abscessus. As a next step, we investigated the assay conditions for infecting silkworms with M. avium, M. intracellulare, M. bovis and My. abscessus. As a result, My. abscessus killed all silkworms efficiently and we established a silkworm infection assay with My. abscessus. Using this infection model, clinically used mycobacterial agents and screened anti-NTM antibiotics were evaluated.

\section{Results}

\subsection{Establishment of a Silkworm Infection Assay with My. abscessus}

Based on the conditions for the silkworm infection assay with M. smegmatis [21], the temperature $\left(37^{\circ} \mathrm{C}\right)$ for breeding silkworms (fifth-instar larvae) and the colony number of the four mycobacteria (M. avium, M. intracellulare, M. bovis and My. abscessus) injected into silkworms were investigated. When the four mycobacteria were injected into silkworms at the highest CFU and the silkworms were bred at $37^{\circ} \mathrm{C}$, only My. abscessus injection $\left(1.5 \times 10^{8} \mathrm{CFU} /\right.$ larva) killed all silkworms $(n=5)$ within $40 \mathrm{~h}$ (Figure 1). Silkworms lived much longer or did not die by injection of the other mycobacteria, including a mixture of M. avium and M. intracellulare. Thus, among the mycobacteria, a silkworm infection assay can be applied for My. abscessus.

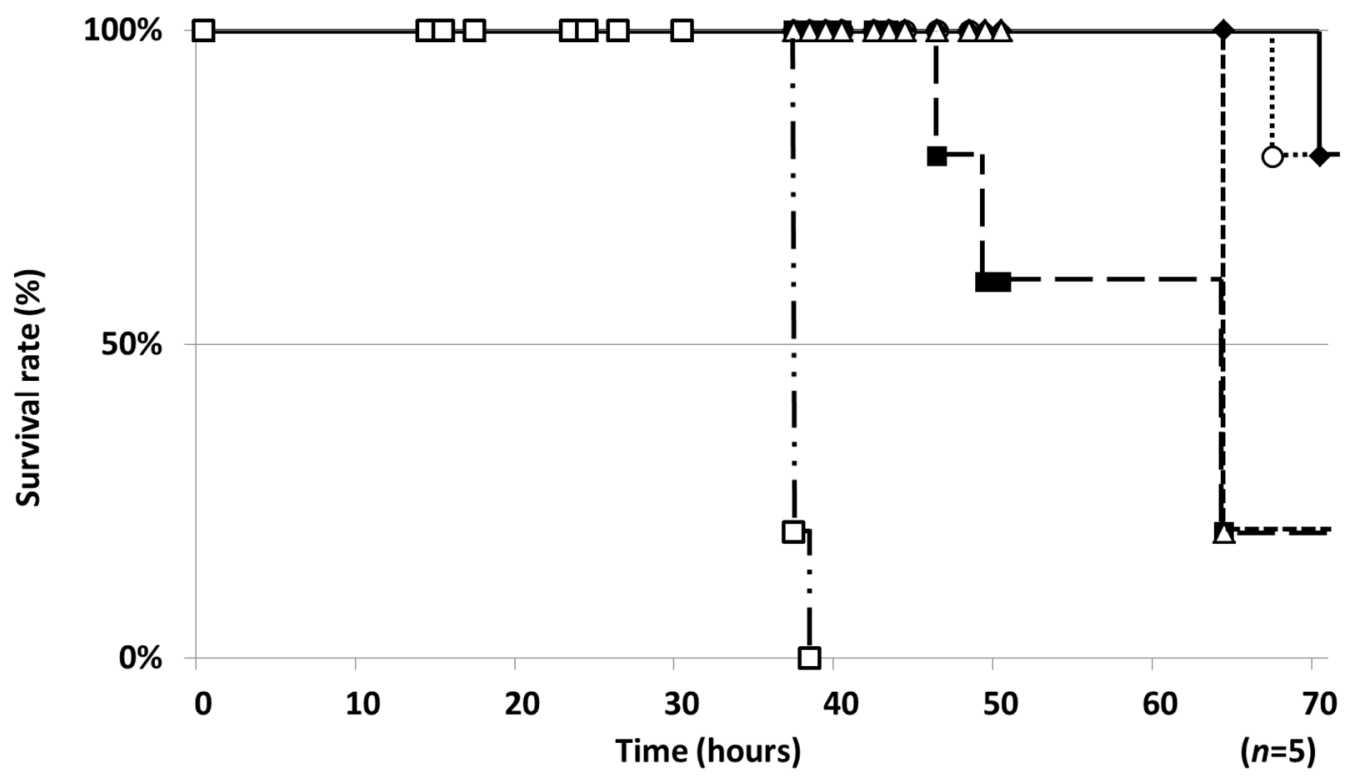

Figure 1. Silkworm-killing ability of four mycobacteria. A suspension of four mycobacteria strains was diluted to the indicated cell number and injected into the silkworm hemolymph. Infected silkworms were incubated at $37{ }^{\circ} \mathrm{C}$. The number of surviving silkworms was counted $70 \mathrm{~h}$ after the injection. ४: M. avium JCM15430 (6.0 × $10^{7} \mathrm{CFU} /$ larva); $\mathbf{~ n : ~ M . ~ i n t r a c e l l u l a r e ~ J C M 6 3 8 4 ~}\left(7.0 \times 10^{8} \mathrm{CFU} /\right.$ larva); $\triangle:$ M. avium + M. intracellulare $\left(1.8 \times 10^{8}+3.5 \times 10^{8} \mathrm{CFU} /\right.$ larva $) ; \bigcirc:$ M. bovis BCG Pasteur $\left(5.0 \times 10^{8}\right.$ CFU/larva); $\square:$ My. abscessus ATCC19977 (1.5 × $10^{8}$ CFU/larva). Experiments were performed three times and reproducible data were observed. 
Next, seven different colony numbers $\left(1.5 \times 10^{6}\right.$ to $\left.1.5 \times 10^{8} \mathrm{CFU} / \mathrm{larva}\right)$ of $\mathrm{My}$. abscessus were subsequently injected into silkworms and infected silkworms were bred at $37^{\circ} \mathrm{C}$. As shown in Figure 2, all silkworms died in a colony number-dependent manner. The times to kill all silkworms were $38\left(1.5 \times 10^{8} \mathrm{CFU} /\right.$ larva injection $), 43\left(7.5 \times 10^{7} \mathrm{CFU} /\right.$ larva $), 49\left(3.7 \times 10^{7} \mathrm{CFU} /\right.$ larva $), 59$ $\left(1.5 \times 10^{7} \mathrm{CFU} /\right.$ larva) and $68 \mathrm{~h}\left(1.5 \times 10^{6} \mathrm{CFU} /\right.$ larva $)$. Based on these results, the conditions for the silkworm infection model with $M y$. abscessus were established: injection at $7.5 \times 10^{7} \mathrm{CFU} /$ larva and breeding at $37^{\circ} \mathrm{C}$. Under these conditions, all silkworms died after $43 \mathrm{~h}$.

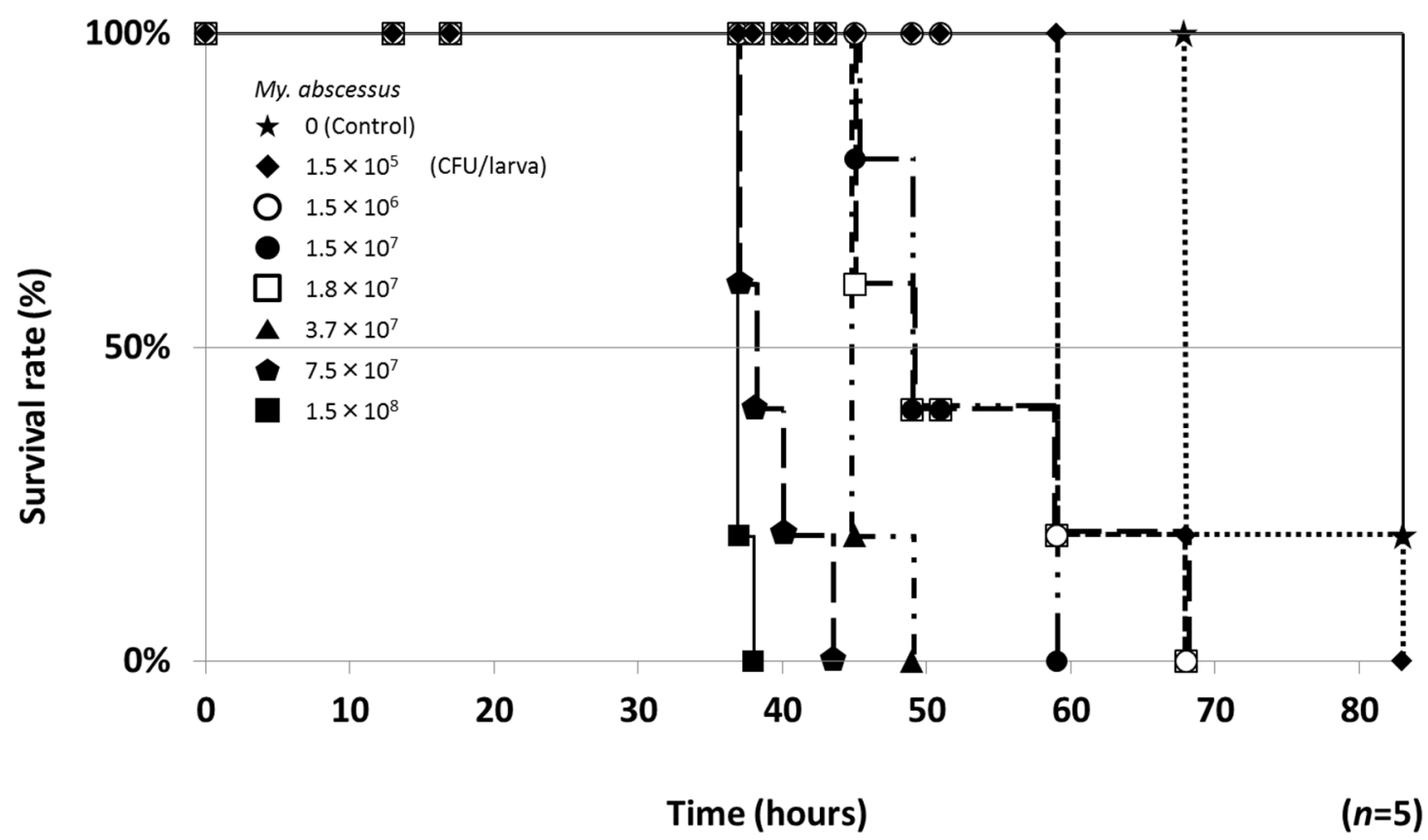

Figure 2. Silkworm-killing ability of My. abscessus. A suspension of My. abscessus ATCC19977 strain was diluted to the indicated cell number and injected into the silkworm hemolymph. Infected silkworms were incubated at $37^{\circ} \mathrm{C}$. The number of surviving silkworms was counted until $83 \mathrm{~h}$ after the injection. Experiments were performed two times and reproducible data were observed.

\subsection{Therapeutic Efficacies of Drugs in the Silkworm Infection Assay with My. abscessus}

Firstly, the toxicity of four clinically used antimicrobial agents (clarithromycin (CAM), amikacin (AMK), imipenem (IPM) and ciprofloxacin (CPFX)) to silkworms was tested (50 $\mu \mathrm{g} / \mathrm{larva}, n=5)$. CAM, AMK and IPM alone exhibited no effect on silkworms. However, CPFX caused silkworm death within $50 \mathrm{~h}$. Then, the four drugs were evaluated in the silkworm infection assay $(n=5)$ with My. abscessus. When CAM, AMK and IPM were injected, silkworms survived in a dose-dependent manner (Figure 3). They exhibited no toxic effects on silkworms at $50 \mu \mathrm{g} / \mathrm{larva}$. On the other hand, CPFX exerted therapeutic effects at 3.12 and $12.5 \mu \mathrm{g} / \mathrm{larva}$, but CPFX was toxic to silkworms at $50 \mu \mathrm{g} / \mathrm{larva}$. Among them, CAM exhibited the strongest therapeutic activity ( $50 \%$ effective dose ( $\left.\left.E D_{50}\right), 0.22 \mu \mathrm{g} / \mathrm{larva}\right)$, followed by AMK $\left(\mathrm{ED}_{50}, 1.48 \mu \mathrm{g} / \mathrm{larva}\right)$. The $\mathrm{ED}_{50}$ values of the four antimicrobial agents against My. abscessus are summarized in Table 2. 


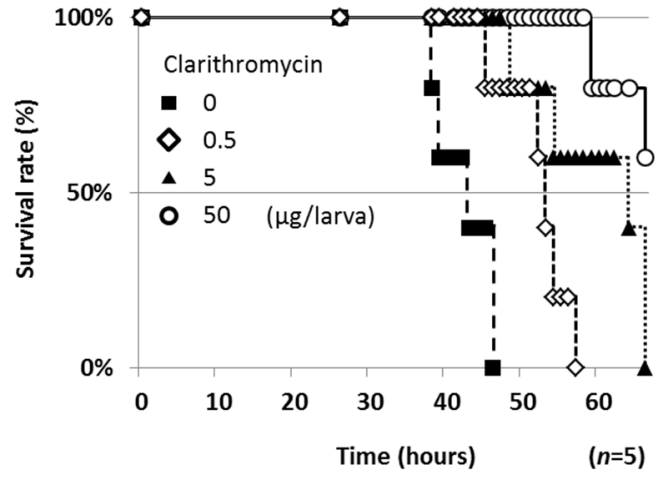

(a)

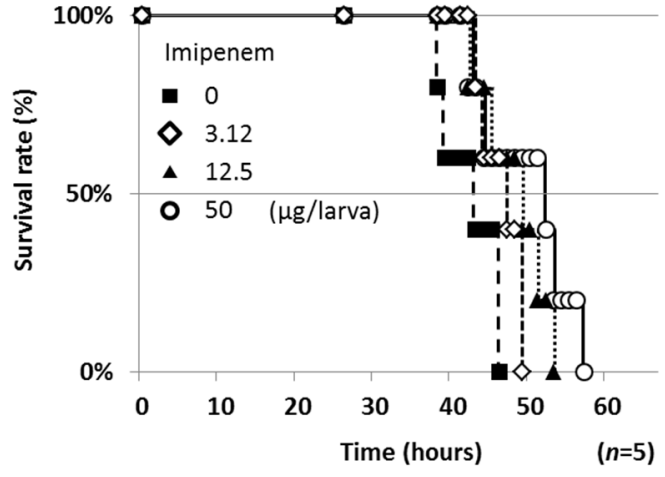

(c)

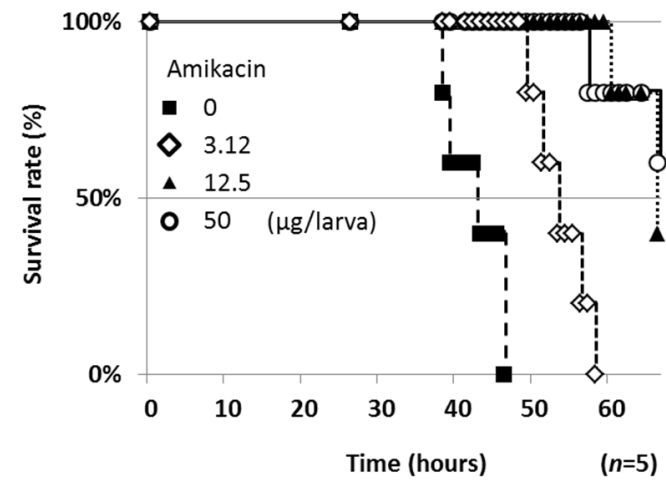

(b)

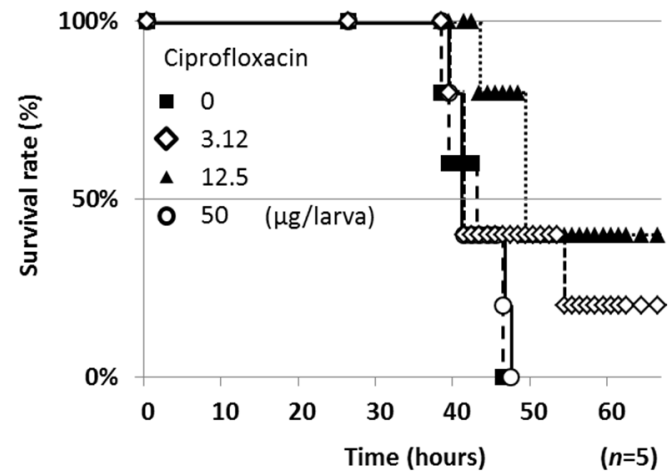

(d)

Figure 3. Therapeutic effects of anti-My. abscessus drugs in the silkworm infection assay with My. abscessus. (a) Clarithromycin, (b) amikacin, (c) imipenem and (d) ciprofloxacin.

\subsection{Screening for Anti-NTM Compounds from a Microbial Product Library}

Our microbial product library consisting of approximately 400 pure compounds was screened for compounds active against all three NTM, M. avium, M. intracellulare and My. abscessus, using the liquid microdilution method [16]. As a result, six microbial compounds (lariatin A (1), nosiheptide (2), ohmyungsamycin A (3), ohmyungsamycin B (4), quinomycin A (5) and steffimycin (6)) (Figure 4) demonstrated potent anti-NTM activity. The minimum inhibitory concentration (MIC) values are summarized in Table 1. Among them, quinomycin A (5) was the most effective against My. abscessus (MIC, $0.01 \mu \mathrm{g} / \mathrm{mL})$, followed by lariatin A (1) (MIC, $1.56 \mu \mathrm{g} / \mathrm{mL})$ and nosiheptide (2) (MIC, $1.56 \mu \mathrm{g} / \mathrm{mL})$. The MICs of CAM, AMK, IPM and CPFX against My. abscessus are also shown in Table 1 for comparative purposes. We demonstrated that compounds 1-6 exhibited anti-My. abscessus activity.

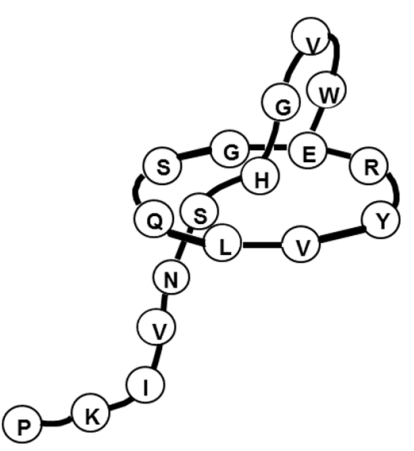

Lariatin 1 (1)

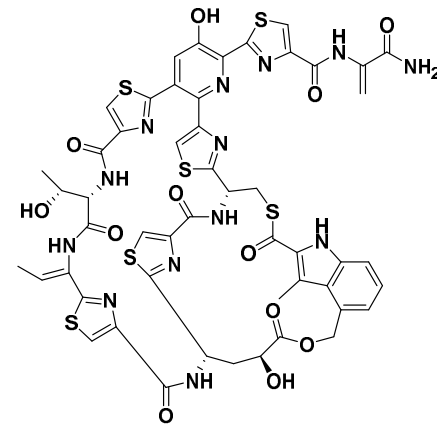

Nosiheptide (2)

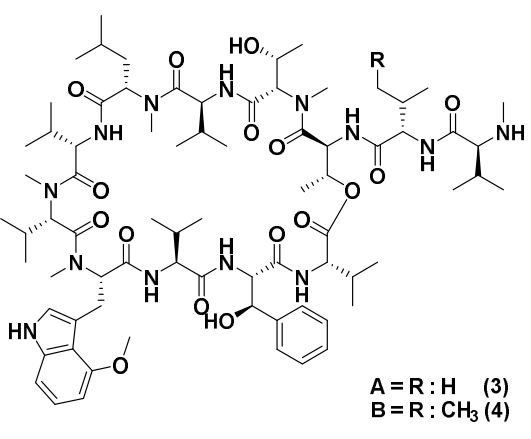

Ohmyungsamycin (3 and 4)

Figure 4. Cont. 


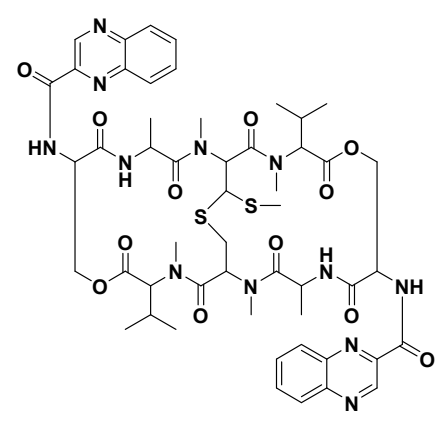

Quinomycin A (5)

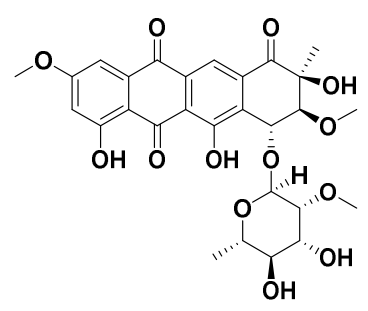

Steffimycin (6)

Figure 4. Structures of microbial anti-non-tuberculous mycobacteria (NTM) compounds.

Table 1. MIC values of anti-NTM agents against four mycobacteria.

\begin{tabular}{ccccccccccc}
\hline \multirow{2}{*}{ Test Microorganism } & \multicolumn{10}{c}{ MIC $(\mu \mathrm{g} / \mathbf{m L}) *$} \\
\cline { 2 - 11 } & $\mathbf{1}$ & $\mathbf{2}$ & $\mathbf{3}$ & $\mathbf{4}$ & $\mathbf{5}$ & $\mathbf{6}$ & $\mathbf{C A M}$ & AMK & IPM & CPFX \\
\hline $\begin{array}{c}\text { M. avium JCM15430 } \\
\text { M. intracellulare }\end{array}$ & 0.78 & 0.02 & 0.19 & 0.19 & $<0.01$ & 1.56 & 0.19 & 3.12 & $>50$ & 0.78 \\
$\quad 1.56$ & 0.02 & 0.10 & 0.10 & $<0.01$ & 0.39 & 0.02 & 0.78 & 1.56 & 0.09 \\
$\quad \begin{array}{c}\text { JCM6384 } \\
\text { M. bovis BCG Pasteur } \\
\quad \text { My. abscessus }\end{array}$ & 0.78 & 0.01 & N.T. & N.T. & N.T. & 1.56 & 0.12 & 0.78 & $>50$ & 0.09 \\
$\quad$ ATCC19977 & 1.56 & 1.56 & 12.5 & 12.5 & 0.01 & 25.0 & 0.39 & 12.5 & 12.5 & 1.56 \\
\hline
\end{tabular}

1: lariatin A, 2: nosiheptide, 3: ohmyungsamycin A, 4: ohmyungsamycin B, 5: quinomycin A, 6: steffimycin, N.T.: not tested. * minimum inhibitory concentration.

\subsection{Therapeutic Efficacies of Microbial Compounds in the Silkworm Infection Assay with My. abscessus}

The study compounds were evaluated in the silkworm infection assay. The survival rate of infected silkworms $(n=5)$ after injection of the study compounds at a dose of $50 \mu \mathrm{g} / \mathrm{larva}$ is shown in Figure 5. Under the conditions in which all infected silkworms (control) died within $48 \mathrm{~h}$, all of the study compounds except 5 prolonged the survival of infected silkworms. In contrast, $\mathbf{5}$, which had the most potent anti-My. abscessus activity in vitro, markedly reduced the survival, probably due to its marked toxic effects on silkworms. Next, the dose dependency of 1-4 was investigated (Figure 6). Compounds $\mathbf{1}$ and $\mathbf{2}$ prolonged the survival in a dose-dependent manner, whereas $\mathbf{3}$ and $\mathbf{4}$ had subtle effects with dose dependency. The $\mathrm{ED}_{50}$ values of these compounds against $\mathrm{My}$. abscessus are summarized in Table 2. Compounds $\mathbf{1}$ and $\mathbf{2}$ exerted therapeutic effects with respective $\mathrm{ED}_{50}$ values of 8.84 and $14.6 \mu \mathrm{g} /$ larva in the silkworm infection assay. All of the study compounds except 5 (50 $\mathrm{\mu g} / \mathrm{larva})$ did not exhibit toxicity against silkworms for at least $48 \mathrm{~h}$. 


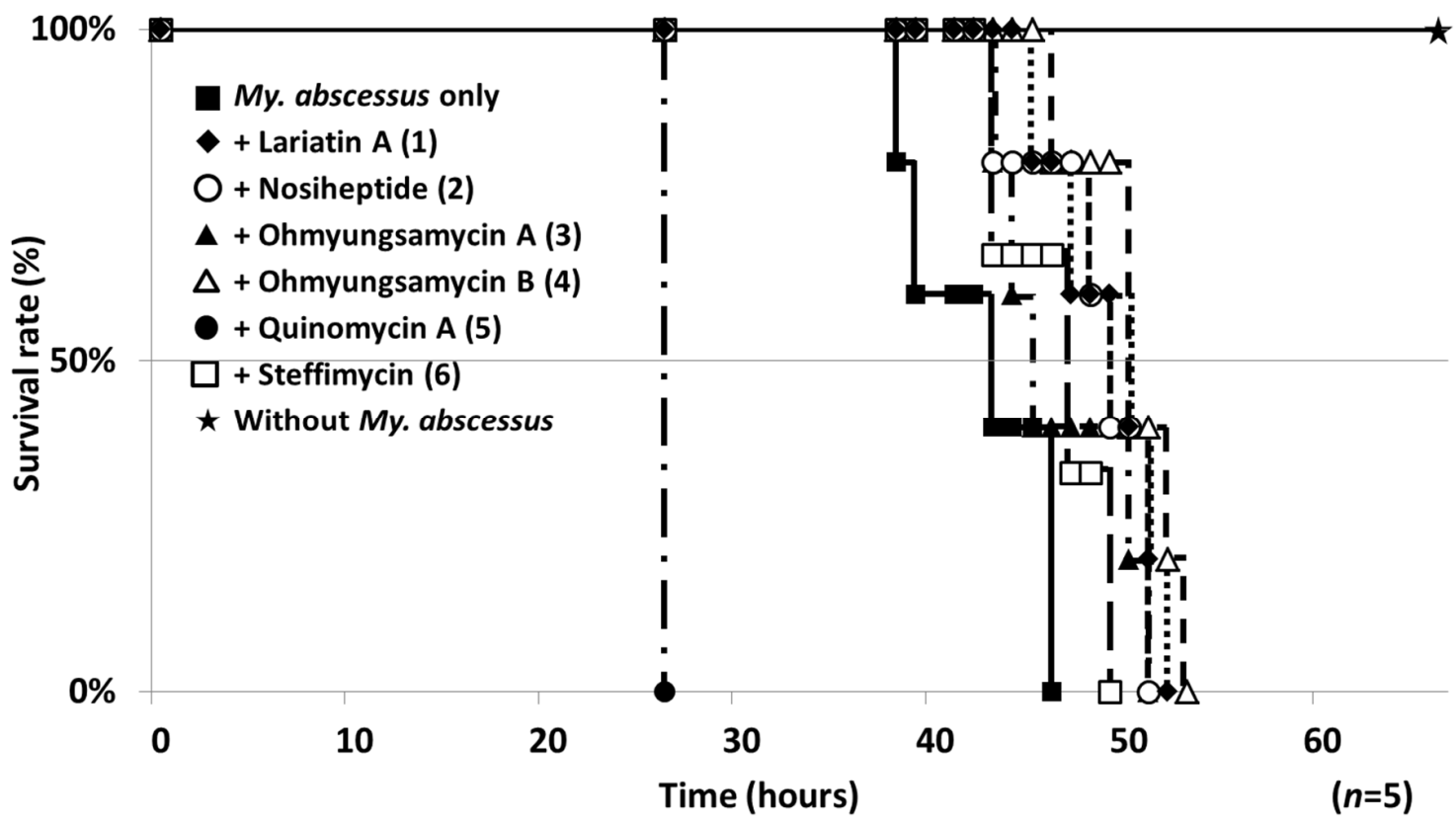

Figure 5. Assay results of microbial anti-NTM compounds in the silkworm infection assay with My. abscessus. The silkworms were injected with My. abscessus $\left(7.5 \times 10^{7} \mathrm{CFU} / \mathrm{larva}\right)$ and the test compound at $50 \mu \mathrm{g} / \mathrm{larva}$.

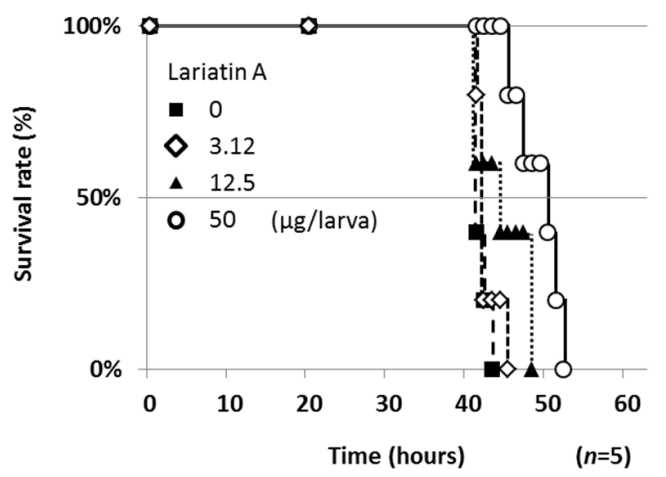

(a)

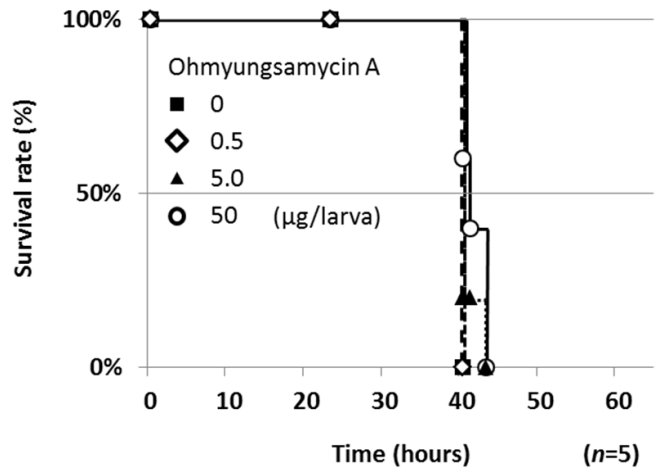

(c)

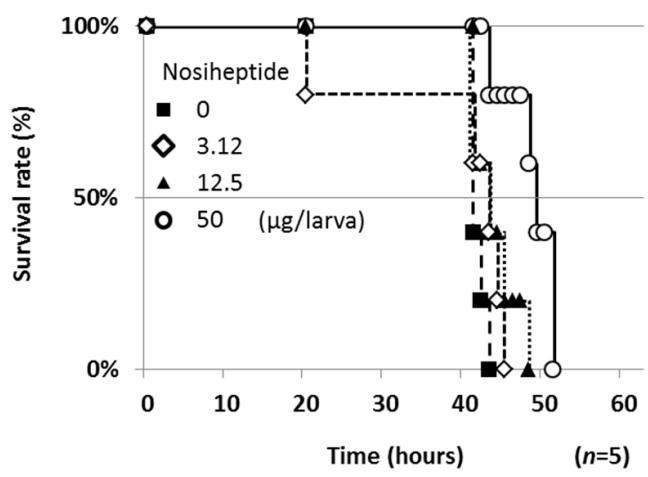

(b)



(d)

Figure 6. Therapeutic effects of microbial anti-NTM compounds in the silkworm infection assay with My. abscessus. (a) Lariatin A (1), (b) nosiheptide (2), (c) ohmyungsamycin A (3) and (d) ohmyungsamycin $\mathrm{B}$ (4). Experiments were performed twice to confirm reproducibility. 
Table 2. MIC and $\mathrm{ED}_{50}$ values of anti-mycobacterial agents against My. abscessus.

\begin{tabular}{cccc}
\hline Test Compound & ED $_{\mathbf{5 0}}(\boldsymbol{\mu g} / \mathbf{l a r v a})^{\mathbf{1}}$ & MIC $(\mu \mathrm{g} / \mathbf{m L})$ & ED $_{\mathbf{5 0}} / \mathbf{M I C}^{-}$ \\
\hline Lariatin A (1) & 8.84 & 1.56 & 5.67 \\
Nosiheptide (2) & 14.6 & 1.56 & 9.36 \\
Ohmyungsamycin A (3) & 30.0 & 12.5 & 2.40 \\
Ohmyungsamycin B (4) & 30.0 & 12.5 & 2.40 \\
Quinomycin A (5) & $>50$ & 25.0 & - \\
Steffimycin (6) & $>50$ & 0.01 & - \\
CAM & 0.22 & 0.39 & 0.56 \\
AMK & 1.48 & 12.5 & 0.12 \\
IPM & 7.82 & 12.5 & 0.63 \\
CPFX & $5.47^{*}$ & 1.56 & 3.51 * \\
\hline
\end{tabular}

1: $50 \%$ effective dose. *: all silkworms died at $50 \mu \mathrm{g} /$ larva.

\section{Discussion}

In the present study, an in vivo-mimic silkworm infection model with four mycobacteria, M. avium, M. intracellulare, M. bovis and My. abscessus, was evaluated. Although the breeding temperature of silkworms $\left(27\right.$ or $\left.37^{\circ} \mathrm{C}\right)$ and the colony number of the mycobacteria for infection were set based on the previous study of a silkworm infection assay with M. smegmatis [21], the silkworms infected with $M$. avium, M. intracellulare and M. bovis did not die within $70 \mathrm{~h}$. Only the silkworms infected with My. abscessus died around $40 \mathrm{~h}$ after infection. M. smegmatis and My. abscessus, rapidly growing mycobacteria, may grow in silkworms, leading to death, whereas $M$. avium, $M$. intracellulare and $M$. bovis, slowly growing mycobacteria, have no ability to kill silkworms. The breeding temperature should be $37^{\circ} \mathrm{C}$ (infected silkworms did not die at $27^{\circ} \mathrm{C}$ ), the best condition for mycobacterial growth, suggesting that the growth speed of mycobacteria is important for this infection assay (Figure 1).

Four clinically used anti-mycobacterial agents were evaluated in this silkworm assay (Figure 3). The order of potency in the in vitro anti-My. abscessus assay (MIC in Table 2) was CAM $>$ CPFX $>$ AMK $=$ IPM, whereas that in the silkworm assay $\left(\mathrm{ED}_{50}\right.$ in Table 2$)$ was $\mathrm{CAM}>\mathrm{AMK}>\mathrm{CPFX}>\mathrm{IPM}$. The orders in both assays are not exactly the same, but they have a similar tendency except for CPFX. CPFX did not exhibit therapeutic efficacy at the highest dose due to its toxicity or insecticidal activity. Sekimizu and colleagues demonstrated that the therapeutic efficacies of clinically used drugs in a silkworm infection assay are consistent with those in a mouse infection assay [7]. Accordingly, the present study suggested the clinical importance of CAM and AMK for the treatment of My. abscessus patients. My. abscessus was reported to have inducible resistance to CAM by the erm(41) gene [22], but it may be difficult to observe such inducible resistance in this silkworm assay because of the short evaluation time (within $70 \mathrm{~h}$ ). There was no preclinical mouse model with My. abscessus. The zebrafish model was generally utilized to evaluate in vivo efficacy until recently [23]. In 2020, Riva et al. reported a new model with immunocompetent mice [24,25]. In this study, we demonstrated the usefulness and effectiveness of silkworm for the first time. Therefore, we consider this silkworm infection model with My. abscessus to be applicable to evaluate the in vivo efficacy of candidate compounds as anti-My. abscessus agents.

From our microbial product library (400 compounds), six compounds exhibited in vitro anti-NTM activity and they were evaluated in this silkworm infection assay with My. abscessus. Lariatin A (1) (produced by Rhodococcus jostii K01-B0171), originally discovered as a selective anti-M. smegmatis antibiotic, also exhibited anti-TB activity [14]. This unique lasso peptide was reported to be effective in the silkworm infection assay with M. smegmatis [21]. In the present study, we demonstrated that 1 was also active against My. abscessus in vitro and in this silkworm infection assay. Nosiheptide was discovered as an anti-Gram-positive antibiotic in 1977 [15]. Recently, we reported that nosiheptide has potent in vitro anti-mycobacterial activity against MAC and M. smegmais. Of note, it was not therapeutically effective in the silkworm infection assay with M. smegmatis [16], but the antibiotic was active in the silkworm infection assay with My. abscessus (Figure 6b). In addition, nosiheptide was reported to have therapeutic effects in a mouse infection assay with Staphylococcus aureus [26]. 
Ohmyungsamycins were discovered as anti-TB antibiotics in $2013[17,27]$, exhibiting weak in vitro and in vivo activity against $M y$. abscessus. Steffimycin, discovered as an anti-cancer antibiotic in 1977 [18,19,28], demonstrated weak in vitro activity against My. abscessus, but no therapeutic effects in the silkworm infection assay with My. abscessus. Quinomycin A, reported as an anti-Gram-positive and anti-cancer antibiotic in 1961 [20,29], exhibited the strongest in vitro activity against My. abscessus, but all silkworms treated with quinomycin A died earlier than infected silkworms (control). Thus, the silkworm infection assay can evaluate not only therapeutic efficacy, but also toxicity. In summary, we demonstrated that microbial compounds 1-6 exhibited anti-My. abscessus activity, and that $\mathbf{1}$ and 2 retained therapeutic efficacy in the silkworm infection assay with My. abscessus. We consider this silkworm infection model to be applicable to the evaluation of the in vivo effectiveness of candidate compounds.

Hamamoto et al. previously reported that the $\mathrm{ED}_{50} / \mathrm{MIC}$ value of a compound is an index of drug potential, and the ratio is typically below 10 for clinically useful antibiotics [7]. As shown in Table 2, the ratios of $\mathbf{1}$ and $\mathbf{2}$ were below 10, suggesting that they are potential anti-My. abscessus drugs.

In conclusion, we established an in vivo-mimic silkworm infection assay with My. abscessus. The therapeutic efficacies of clinically used anti-NTM drugs and microbial compounds were evaluated in this silkworm infection assay, suggesting their potential in vivo efficacies. As chemotherapeutic drugs for the treatment of $M y$. abscessus patients are limited, this silkworm infection model will be valuable to select practically effective anti-My. abscessus drug candidates.

\section{Materials and Methods}

\subsection{Materials}

Lariatin A, nosiheptide, ohmyungsamycins, steffimycin and quinomycin A were purified from a culture broth of actinomycetes in our laboratory. Clarithromycin (CAM), amikacin (AMK), imipenem (IPM) and ciprofloxacin (CPFX) were purchased from Wako Pure Chemical Industries (Osaka, Japan). Middlebrook 7H9 broth (Becton, Dickinson and Company, Franklin Lakes, NJ, USA) containing 0.05\% Tween 80 (Tokyo Chemical Industries: Tokyo, Japan) and 10\% albumin dextrose catalase (ADC) enrichment ( $5 \%$ bovine serum albumin, Sigma Aldrich (MO, USA); $2 \%$ glucose, Wako Pure Chemical Industries (Osaka, Japan); $0.85 \% \mathrm{NaCl}$, Wako Pure Chemical Industries) was used for the cultivation of mycobacteria. Third-molting larvae stage silkworms, Bombyx mori (Hu^yo $\times$ Tukuba $\bullet$ Ne), were purchased from Ehime Sanshu (Ehime, Japan). Silk Mate 2S, an artificial diet containing antibiotics, was purchased from Nosan Corporation (Kanagawa, Japan). The following mycobacterial strains were used in this study: M. avium JCM15430, M. intracellulare JCM6384, M. bovis BCG Pasteur and My. abscessus ATCC19977. The M. bovis BCG Pasteur used was a laboratory strain. M. avium JCM15430 and M. intracellulare JCM6384 were purchased from the Riken BioResource Research Center (Ibaraki, Japan). My. abscessus ATCC19977 was purchased from the American Type Culture Collection (VA, USA).

\subsection{Preparation of Mycobacterial Suspension}

M. avium JCM15430, M. intracellulare JCM6384, M. bovis BCG Pasteur and My. abscessus ATCC19977 were stored in $20 \%$ glycerol at $-80{ }^{\circ} \mathrm{C}$. The frozen stock cultures of $M$. avium, M. intracellulare, $M$. bovis and My. abscessus (200, 120, 500 and $200 \mu \mathrm{L})$ were inoculated in Middlebrook 7H9 broth $(6 \mathrm{~mL})$ in a T-25 flask (Corning, Corning, NY, USA), and cultured under static conditions at $37^{\circ} \mathrm{C}$ for $96,72,96$ and $48 \mathrm{~h}$, respectively $\left(>1 \times 10^{8} \mathrm{CFU} / \mathrm{mL}\right)$.

\subsection{MIC Values in the Liquid Microdilution Assay}

Anti-mycobacterial activities against these four strains were evaluated according to a previously established liquid microdilution method [16]. 
M. avium, M. intracellulare, $M$. bovis and My. abscessus suspensions were adjusted to $4.0 \times 10^{6}-1 \times 10^{7} \mathrm{CFU} / \mathrm{mL}$ in Middlebrook $7 \mathrm{H} 9$ broth containing $0.05 \%$ Tween 80 and $10 \%$ ADC enrichment. The suspension $(95 \mu \mathrm{L})$ was added to each well of a 96-well microplate (Corning) with or without the test drugs ( $5 \mu \mathrm{L}$ in $\mathrm{MeOH}$ or water) and incubated at $37^{\circ} \mathrm{C}$ for $72-120 \mathrm{~h}$. MTT reagent $(5.5 \mathrm{mg} / \mathrm{mL}$ MTT, $5 \mu \mathrm{L})$ was added to each well and the cells were incubated for $16 \mathrm{~h}$. After cells were lysed with lysis buffer (40\% N,N-dimethylformamide, Nacalai Tesque, Kyoto, Japan; 20\% SDS, Wako Pure Chemical Industries; $2 \% \mathrm{CH}_{3} \mathrm{COOH}$, Kanto Chemical, Tokyo, Japan; $95 \mu \mathrm{L}$ ), the absorbance of the lysate was measured at $570 \mathrm{~nm}$ using an absorption spectrometer. The MIC value was defined as the lowest drug concentration that resulted in $90 \%$ growth inhibition of M. avium, M. intracellulare, M. bovis and My. abscessus.

\subsection{Silkworm Infection Assay with Mycobacteria Spp.}

Hatched silkworm larvae were raised by feeding an artificial diet containing antibiotics (Silk Mate 2S) in an incubator at $27^{\circ} \mathrm{C}$ until the fourth molting stage. On the first day of fifth-instar larvae, silkworms $(n=5)$ were fed Silk Mate 2S. On the second day, mycobacterial suspensions $\left(6.0 \times 10^{7}\right.$ to $7.0 \times 10^{8} \mathrm{CFU} /$ larva $)$ were injected into the hemolymph through the dorsal surface of the silkworm using a disposable 1-mL syringe with a 27-G needle (TERUMO, Tokyo, Japan). After injection, the number of silkworms that survived was counted at the indicated time until $80 \mathrm{~h}$. The data are plotted according to the Kaplan-Meier method [30].

\subsection{Silkworm Infection Assay with My. abscessus}

Seven different cell numbers $\left(1.5 \times 10^{6}\right.$ to $\left.1.5 \times 10^{8} \mathrm{CFU} / \mathrm{larva}\right)$ of $\mathrm{My}$. abscessus suspensions were subsequently injected into the hemolymph through the dorsal surface of the silkworm $(2.0 \mathrm{~g}, n=5)$ using a disposable 1-mL syringe with a $27-\mathrm{G}$ needle, and the number of silkworms that survived was counted at the indicated time until $80 \mathrm{~h}$. The data are plotted according to the Kaplan-Meier method [30]

\subsection{ED 50 Values in the Silkworm Infection Assay with My. abscessus}

A My. abscessus ATCC19977 suspension (7.5 × 107 CFU/larva in $50 \mu$ L Middlebrook 7H9 broth) was injected into the hemolymph of silkworm larvae $(2.0 \mathrm{~g}, n=5)$, followed by the injection of anti-My. abscessus drugs or microbial compounds (50 $\mu \mathrm{L}$ in water or $10 \%$ DMSO) within 30 min. Silkworms were maintained at $37^{\circ} \mathrm{C}$. The dose of a sample leading to a $50 \%$ survival rate $\left(\mathrm{ED}_{50}\right)$ was calculated at the time when all My. abscessus-infected silkworms without sample injection died (around $43 \mathrm{~h})$, according to a previous method [7,21,31].

Author Contributions: N.K., R.U., H.H. and H.T. designed the study. A.K. provided microorganisms for screening. K.H. and A.Y. performed the experiments. K.H. and H.T. wrote the paper. All authors have read and agreed to the published version of the manuscript.

Funding: This study was partially supported by JSPS KAKENHI Grant Numbers 21310146 (HT), 15 K07867 (NK) and 19J15511 (AY) from the Ministry of Education, Culture, Sports, Science and Technology, Japan.

Conflicts of Interest: The authors declare no conflict of interest.

\section{References}

1. Hamamoto, H.; Urai, M.; Ishii, K.; Yasukawa, J.; Paudel, A.; Murai, M.; Kaji, T.; Kuranaga, T.; Hamase, K.; Katsu, T.; et al. Lysocin E is a new antibiotic that targets menaquinone in the bacterial membrane. Nat. Chem. Biol. 2015, 11, 127-133. [CrossRef] [PubMed]

2. Uchida, R.; Iwatsuki, M.; Kim, Y.P.; Ohte, S.; Omura, S.; Tomoda, H. Nosokomycins, new antibiotics discovered in an in vivo-mimic infection model using silkworm larvae. I: Fermentation, isolation and biological properties. J. Antibiot. (Tokyo) 2010, 63, 151-155. [CrossRef] [PubMed] 
3. Uchida, R.; Iwatsuki, M.; Kim, Y.P.; Omura, S.; Tomoda, H. Nosokomycins, new antibiotics discovered in an in vivo-mimic infection model using silkworm larvae. II: Structure elucidation. J. Antibiot. (Tokyo) 2010, 63, 157-163. [CrossRef] [PubMed]

4. Uchida, R.; Hanaki, H.; Matsui, H.; Hamamoto, H.; Sekimizu, K.; Iwatsuki, M.; Kim, Y.P.; Tomoda, H. In vitro and in vivo anti-MRSA activities of nosokomycins. Drug Discov. Ther. 2014, 8, 249-254. [CrossRef] [PubMed]

5. Uchida, R.; Namiguchi, S.; Ishijima, H.; Tomoda, H. Therapeutic effects of three trichothecenes in the silkworm infection assay with Candida albicans. Drug Discov. Ther. 2016, 10, 44-48. [CrossRef] [PubMed]

6. Kaito, C.; Akimitsu, N.; Watanabe, H.; Sekimizu, K. Silkworm larvae as an animal model of bacterial infection pathogenic to humans. Microb. Pathog. 2002, 32, 183-190. [CrossRef] [PubMed]

7. Hamamoto, H.; Kurokawa, K.; Kaito, C.; Kamura, K.; Manitra Razanajatovo, I.; Kusuhara, H.; Santa, T.; Sekimizu, K. Quantitative evaluation of the therapeutic effects of antibiotics using silkworms infected with human pathogenic microorganisms. Antimicrob. Agents. Chemother. 2004, 48, 774-779. [CrossRef]

8. Matsumoto, Y.; Miyazaki, S.; Fukunaga, D.H.; Shimizu, K.; Kawamoto, S.; Sekimizu, K. Quantitative evaluation of cryptococcal pathogenesis and antifungal drugs using a silkworm infection model with Cryptococcus neoformans. J. Appl. Microbiol. 2012, 112, 138-146. [CrossRef]

9. Adjemian, J.; Olivier, K.N.; Seitz, A.E.; Holland, S.M.; Prevots, D.R. Prevalence of nontuberculous mycobacterial lung disease in U.S. Medicare beneficiaries. Am. J. Respir. Crit. Care. Med. 2012, 185, 881-886. [CrossRef]

10. Marras, T.K.; Mendelson, D.; Marchand-Austin, A.; May, K.; Jamieson, F.B. Pulmonary nontuberculous mycobacterial disease, Ontario, Canada, 1998-2010. Emerg. Infect. Dis. 2013, 19, 1889-1891. [CrossRef]

11. Namkoong, H.; Kurashima, A.; Morimoto, K.; Hoshino, Y.; Hasegawa, N.; Ato, M.; Mitarai, S. Epidemiology of Pulmonary Nontuberculous Mycobacterial Disease, Japan. Emerg. Infect. Dis. 2016, 22, 1116-1117. [CrossRef] [PubMed]

12. Griffith, D.E.; Aksamit, T.; Brown-Elliott, B.A.; Catanzaro, A.; Daley, C.; Gordin, F.; Holland, S.M.; Horsburgh, R.; Huitt, G.; Iademarco, M.F.; et al. An official ATS/IDSA statement: Diagnosis, treatment, and prevention of nontuberculous mycobacterial diseases. Am. J. Respir. Crit. Care Med. 2007, 175, 367-416. [CrossRef] [PubMed]

13. Adelman, M.H.; Addrizzo-Harris, D.J. Management of nontuberculous mycobacterial pulmonary disease. Curr. Opin. Pulm. Med. 2018, 24, 212-219. [CrossRef]

14. Iwatsuki, M.; Tomoda, H.; Uchida, R.; Gouda, H.; Hirono, S.; Omura, S. Lariatins, antimycobacterial peptides produced by Rhodococcus sp. K01-B0171, have a lasso structure. J. Am. Chem. Soc. 2006, 128, 7486-7491. [CrossRef] [PubMed]

15. Pascard, C.; Ducruix, A.; Lunel, J.; Prang, T. Highly modified cysteine-containing antibiotics. Chemical structure and configuration of nosiheptide. J. Am. Chem. Soc. 1977, 99, 6418-6423. [CrossRef] [PubMed]

16. Hosoda, K.; Koyama, N.; Kanamoto, A.; Tomoda, H. Discovery of nosiheptide, griseoviridin, and etamycin as potent anti-mycobacterial agents against Mycobacterium avium complex. Molecules 2019, 24, 1495. [CrossRef] [PubMed]

17. Um, S.; Choi, T.J.; Kim, H.; Kim, B.Y.; Kim, S.H.; Lee, S.K.; Oh, K.B.; Shin, J.; Oh, D.C. Ohmyungsamycins A and B: Cytotoxic and antimicrobial cyclic peptides produced by Streptomyces sp. from a volcanic island. J. Org. Chem. 2013, 78, 12321-12329. [CrossRef]

18. Bergy, M.E.; Reusser, F. A new antibacterial agent (U-20,661) isolated from a Streptomycete strain. Experientia 1967, 23, 254-255. [CrossRef]

19. Koyama, N.; Shigeno, S.; Kanamoto, A.; Tomoda, H. Steffimycin E, a new anti-mycobacterial agent against Mycobacterium avium complex, produced by Streptomyces sp. OPMA02852. J. Antibiot. (Tokyo) 2020, 73, 581-584. [CrossRef]

20. Yoshida, T.; Katagiri, K.; Yokozawa, S. Studies on quinoxaline antibiotics. II. Isolation and properties of quinomycins A, B and C. J. Antibiot. (Tokyo) 1961, 14, 330-334.

21. Yagi, A.; Uchida, R.; Hamamoto, H.; Sekimizu, K.; Kimura, K.I.; Tomoda, H. Anti-Mycobacterium activity of microbial peptides in a silkworm infection model with Mycobacterium smegmatis. J. Antibiot. (Tokyo) 2017, 70, 685-690. [CrossRef] [PubMed]

22. Nash, K.A.; Brown-Elliott, B.A.; Wallace, R.J. A novel gene, erm(41), confers inducible macrolide resistance to clinical isolates of Mycobacterium abscessus but is absent from Mycobacterium chelonae. Antimicrob. Agents Chemother. 2009, 53, 1367-1376. [CrossRef] 
23. Dupont, C.; Viljoen, A.; Dubar, F.; Blaise, M.; Bernut, A.; Pawlik, A.; Bouchier, C.; Brosch, R.; Guèrardel, Y.; Lelièvre, J.; et al. A new piperidinol derivative targeting mycolic acid transport in Mycobacterium abscessus. Mol. Microbiol. 2016, 101, 515-529. [CrossRef] [PubMed]

24. Riva, C.; Tortoli, E.; Cugnata, F.; Sanvito, F.; Esposito, A.; Rossi, M.; Colarieti, A.; Canu, T.; Cigana, C.; Bragonzi, A.; et al. A New Model of Chronic Mycobacterium abscessus Lung Infection in Immunocompetent Mice. Int. J. Mol. Sci. 2020, 21, 6590. [CrossRef]

25. Maggioncalda, E.C.; Story-Roller, E.; Mylius, J.; Illei, P.; Basaraba, R.J.; Lamichhane, G. A mouse model of pulmonary Mycobacteroides abscessus infection. Sci. Rep. 2020, 10, 3690. [CrossRef] [PubMed]

26. Haste, N.M.; Thienphrapa, W.; Tran, D.N.; Loesgen, S.; Sun, P.; Nam, S.J.; Jensen, P.R.; Fenical, W.; Sakoulas, G.; Nizet, V.; et al. Activity of the thiopeptide antibiotic nosiheptide against contemporary strains of methicillin-resistant Staphylococcus aureus. J. Antibiot. (Tokyo) 2012, 65, 593-598. [CrossRef] [PubMed]

27. Kim, T.S.; Shin, Y.H.; Lee, H.M.; Kim, J.K.; Choe, J.H.; Jang, J.C.; Um, S.; Jin, H.S.; Komatsu, M.; Cha, G.H.; et al. Ohmyungsamycins promote antimicrobial responses through autophagy activation via AMP-activated protein kinase pathway. Sci. Rep. 2017, 7, 3431. [CrossRef] [PubMed]

28. Reusser, F. Mode of action of antibiotic U-20,661. J. Bacteriol. 1967, 93, 65-70. [CrossRef]

29. Sato, K.; Yoshida, T.; Katagiri, K. Inhibition of RNA synthesis in E. coli protoplasts by quinomycins A and C. J. Antibiot. (Tokyo) 1967, 20, 188-189.

30. Kaplan, E.; Meier, P. Nonparametric Estimation from Incomplete Observations. J. Am. Stat. Assoc. 1958, 53, 457-481. [CrossRef]

31. Tominaga, T.; Uchida, R.; Koyama, N.; Tomoda, H. Anti-Rhizopus activity of tanzawaic acids produced by the hot spring-derived fungus Penicillium sp. BF-0005. J. Antibiot. (Tokyo) 2018, 71, 626-632. [CrossRef] [PubMed]

Sample Availability: Samples of compounds 1-6 are available from the authors.

Publisher's Note: MDPI stays neutral with regard to jurisdictional claims in published maps and institutional affiliations.

(C) 2020 by the authors. Licensee MDPI, Basel, Switzerland. This article is an open access article distributed under the terms and conditions of the Creative Commons Attribution (CC BY) license (http://creativecommons.org/licenses/by/4.0/). 